\title{
Can you hear me now? A genetic model of otitis media with effusion
}

\author{
Evelyn Lazaridis and James C. Saunders
}

Department of Otorhinolaryngology, Head and Neck Surgery, University of Pennsylvania, Philadelphia, Pennsylvania, USA.

\begin{abstract}
Otitis media with effusion (OME) is characterized by the occurrence of fluid in the middle-ear cavity in the absence of any signs of acute ear infection and occurs most frequently in children with auditory or eustachian tube dysfunction. Its chronic form is an important clinical issue for pediatricians and otologists alike. The study by Depreux et al. in this issue of the JCI shows that absence of the transcriptional activator Eya4 in knockout mice results in abnormal structuring of the eustachian tube, thus predisposing these animals to OME (see the related article beginning on page 651). The development of this genetics-based animal model is an important advance for understanding OME and for exploring new avenues of treatment.
\end{abstract}

In humans, fluid accumulation in the middle-ear cavity, in the absence of acute ear infection, is known as otitis media with effusion (OME) (1-3). The disorder occurs most frequently in children, with approximately 2.2 million episodes diagnosed each year in the United States $(2,3)$. Fortunately, spontaneous resolution occurs within three months in a large number of these children. Unfortunately, an equally large number of children have recurrent OME (1). Recurrent OME is accompanied by an increased risk for sustained conductive hearing loss, with the potential for speech, language, and learning problems. The treatment of chronic OME is thus an important clinical issue for pediatricians and otologists.

The most important factor associated with OME is abnormal structure and function of the eustachian tube (ET) (3). The ET is an osteocartilaginous tube that connects the middle-ear cavity to the nasopharynx. The cartilaginous portion opens into the nasopharynx at the torus tubarius, and the osseous portion opens into the anterior wall of the middle-ear cavity at the ostium (3) (Figure 1A). The lumen of the ET and the inner surface of the middle-ear cavity are lined with the respiratory epithelium. The mucosal layer of the respiratory epithelium consists of secretory cells, ciliated cells, supporting cells, and connective tissue (3).

Nonstandard abbreviations used: ET, eustachian tube; Eya4, eyes absent 4 homolog; OME, otitis media with effusion.

Conflict of interest: The authors have declared that no conflict of interest exists.

Citation for this article: J. Clin. Invest. 118:471-474 (2008). doi:10.1172/JCI33716.
The ET serves two major functions. First, it permits air to enter or leave the middle-ear cavity, thus balancing middle-ear pressure with ambient air pressure (4). The tensor veli palatini muscle is primarily responsible for active dilatation of the ET, and pressure equilibration occurs most frequently during swallowing. Second, the ET provides a conduit through which middle-ear secretions can be swept into the nasopharynx, via the beating action of the ciliated cells. Secretions in the middle ear can arise from two sources: capillary effusion (also called transudate effusions; ref. 3), and mucus secretory cells of the respiratory epithelium. Herein, we refer to these secretions collectively as middle-ear secretions. In addition, reflux through the ET can draw nasopharynx secretions into the middle-ear cavity.

\section{Human OME}

Immature development of the ET impairs the venting function and increases the likelihood of secretion accumulation in the middle-ear cavity. Other impairments to the ET can also cause accumulation of these secretions. At the torus tubarius, for example, ET obstruction can occur from surrounding epithelium (Figure 1, A and B). At the ostium, the presence of a cholesteatoma (a benign skin growth) or a polyp may block the opening of the ET (Figure 1C). In addition, ET dysfunction can be caused by failure of the tube to close, a flaccid tube, or an inflamed epithelium (Figure 1D). The net effect of all these examples is blockage of the flow of secretions from the middlereleased into the middle-ear cavity from the hypertrophied adenoids or edema of the ear cavity. Impairment of cavity ventilation and drainage is the primary mechanism that triggers an OME episode. In addition, the clearance of middle-ear secretions depends on the integrity of the respiratory epithelium, in particular, the effectiveness of the ciliated cells in moving secretions. Furthermore, craniofacial abnormalities, of which a palatal defect is one example, are also related to a higher incidence of OME because they impair the ET venting mechanism (3).

When middle-ear ventilation is impaired, negative middle-ear pressure results, causing tympanic membrane retraction and the accumulation of middle-ear secretions. Notably, the longer these secretions remain in the cavity, the more likely it is that they will produce structural alterations such as polyp formation, tissue fibrosis, additional ET obstruction, and, eventually, chronic OME (with the so-called "glue ear" condition; ref. 3). The majority of cases of OME are asymptomatic, but in some children, mild intermittent ear pain, fullness, irritability, ear rubbing, balance problems, a conductive hearing loss of 25-40 decibels (dB), delayed language or speech development, and/or problems with school performance may emerge (1). Spontaneous resolution of OME is largely determined by the original cause and the duration of fluid in the middle ear but most frequently occurs within three months. The only effective treatment for chronic OME at present is a tympanostomy with tube insertion (1). This limited option for clinically treating chronic OME, and the difficulty in identifying new treatment procedures, due to the requirement for large studies involving children who are at risk for structural damage to the ear, calls attention to the need for an animal model in which new treatments for OME can be tested.

\section{A genetic basis for OME}

Recently, a new transcriptional activator was identified in the vertebrate eyes absent gene family, called eyes absent bomolog 4 (EYA4) (5). This transcriptional activator plays a developmental role in the early 
A

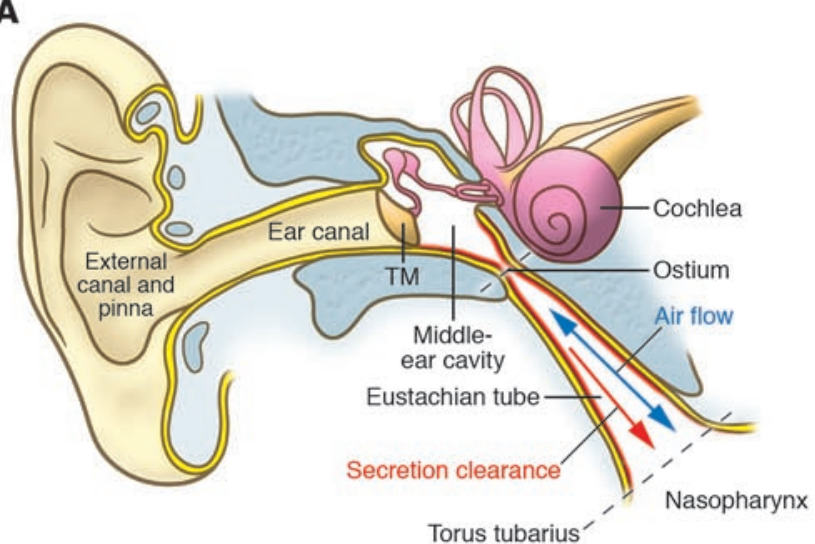

C

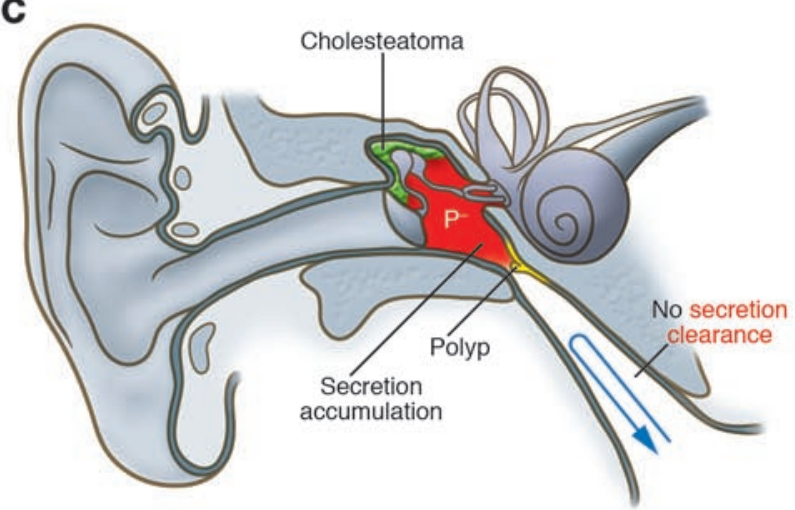

B

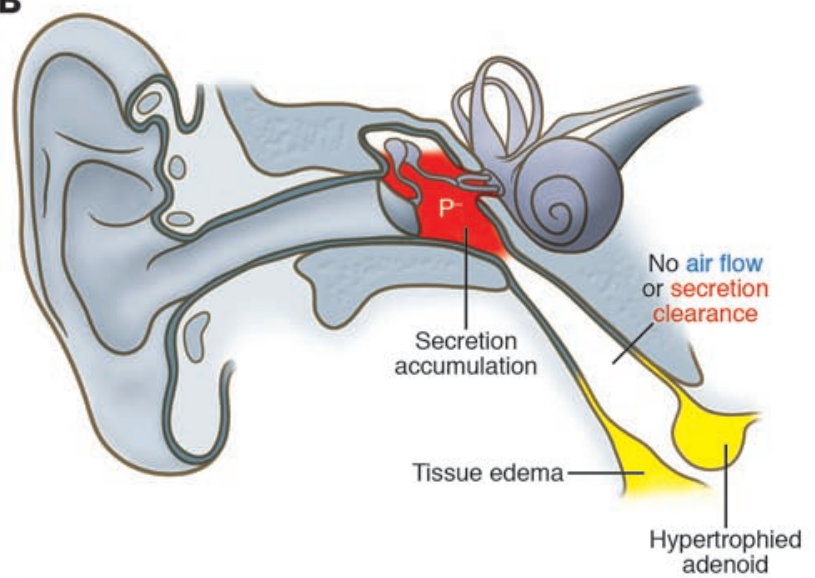

D

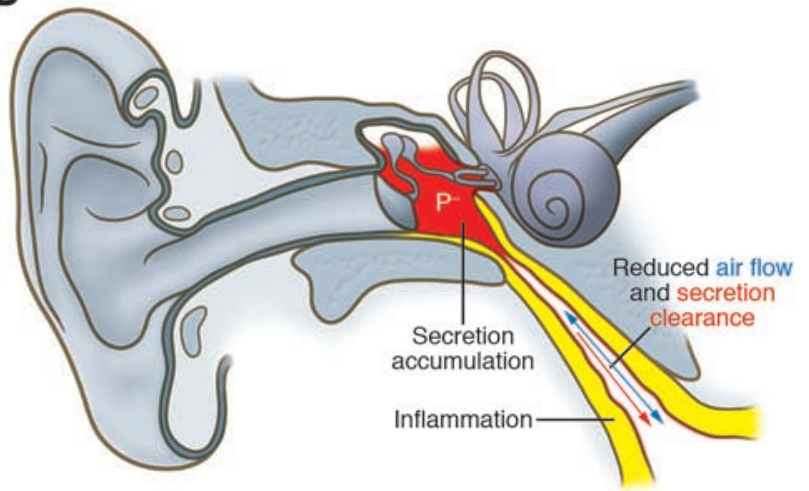

Figure 1

ET physiology. (A) The normal ET. The ET, which joins the nasopharynx at the torus tubarius and joins the middle-ear cavity at the ostium, permits airflow between the middle-ear cavity and nasopharynx, balancing middle-ear pressure with ambient air pressure. Also, middle-ear secretions are swept into the nasopharynx (clearance) from the middle-ear cavity. (B) Mechanical obstruction of the ET may occur at the torus tubarius by adenoid hypertrophy or edema (B), or at the ostium in the middle-ear cavity by perhaps a polyp or a cholesteatoma (C). The pathologies shown in $\mathbf{B}$ or $\mathbf{C}$ block air flow and the clearance of middle-ear cavity secretions though the $\mathrm{ET}$, increasing middle-ear negative pressure $\left(\mathrm{P}^{-}\right)$and accumulation of middle-ear secretions. An intrinsic ET obstruction may also result from epithelium inflammation (D), secondary to nasal infection or allergy. This also results in middle-ear negative pressure and secretion accumulation. TM, tympanic membrane.

stages of normal embryogenesis, as it interacts with proteins in the regulatory hierarchy. In addition, it is also important in the development of structures within the inner ear, such as the cochlear capsule, Reissner membrane, stria vascularis, and the sensory epithelia of the vestibular system. EYA4 mutations in humans are related to postlingual, autosomal dominant, progressive hearing loss at the DFNA10 locus (5).

Eya4-deficient mice (Eya4-/- mice) appear to primarily suffer from disrupted ET morphology. It may be the case that EYA4 mutations in humans result in abnormal anatomy in the middle ear that contributes to the formation of a cholesteatoma. Unfortunately, data to support this possibility have yet to be reported. As reported in this issue of the JCI, Depreux et al. (6), in the process of investigating hearing loss in Eya $4^{-/-}$mice, found that these mutant ani- mals had OME. The existence of a genetic basis for OME has been previously suggested, as the incidence of OME has been shown to be higher in twin siblings (3); but in their current study Depreux et al. propose that deletion of a specific gene, Eya4, causes OME (6). Auditory brainstem responses of the Eya $4^{-/-}$mice studied revealed a substantial loss in peripheral auditory function; however, how much of this was attributable to the middle-ear conductive system is unknown. The investigators also excluded the possibility of their results arising from middle-ear or ET infection by treating pups with antibiotic prophylaxis. The lumen and epithelium of the middle-ear cavity, ET, and craniofacial and palate skeleton were examined in detail to characterize further anatomical defects in the Eya4 ${ }^{-/-}$mice. In comparison to WT mice, the ET ostium in Eya4 ${ }^{-/-}$animals was narrower and abnormally positioned in the middle ear, and $10 \%$ of mutant animals had a mechanical obstruction of the ostium by a polyp (Figure 2). The medial osseous segment was also abnormally narrow in Eya4 $4^{-/}$mice compared with WT mice. Furthermore, there was delayed palatal maturation, which was resolved within four weeks, and cilia cell density in the middle-ear cavity was reduced after 16 months in Eya4 ${ }^{-/-}$mice. Table 1 compares and contrasts the phenotypes of Eya4 ${ }^{-/-}$mice and human infants with OME, revealing that many of the major characteristics of OME in children and the Eya4 $4^{-/-}$mutant mice are the same. Indeed, we suspect that the characteristics of the ET in Eya4 ${ }^{-/-}$mice are almost identical to the immaturity of the ET observed in infants with OME.

The study by Depreux et al. (6) shows that the Eya4 gene may play a critical role 
A WT mice

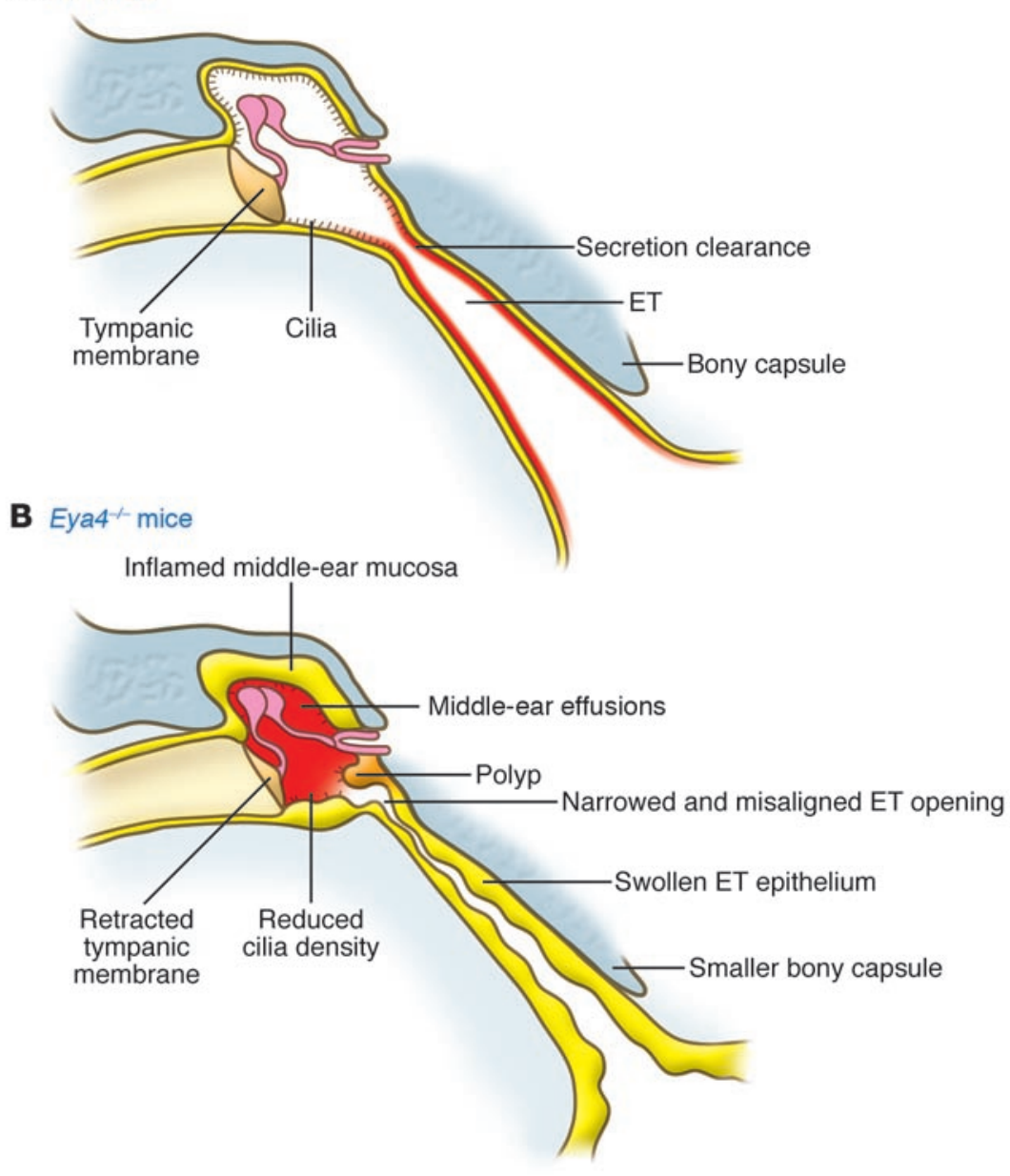

in structuring the ET and that immature development of these structures predisposes Eya $4^{-/-}$mice to OME. While the degree of conductive hearing loss in the Eya4-/mouse is unknown, and may be overshadowed by inner-ear defects, the degree of similarity between the OME dysmorphology of the middle ear observed in the mutant mouse and that observed in humans is nevertheless remarkable. Even those features with delayed onset are similar in both. This correspondence supports the authors' conclusions that the Eyat $4^{-/}$mouse is an excellent genetic model of human OME.

Future studies may reveal other genes whose interactions with Eya4 result in hearing loss and OME, and the identification of such genes could provide a better understanding of OME and aid the development of therapies.

\section{Figure 2}

Dysmorphology of the ear in Eya4 $4^{-/-}$mice is characteristic of human OME. (A) Schematic of normal morphology of the middle-ear cavity and ET in WT mice. (B) In this issue of the $\mathrm{JCl}$, Depreux et al. (6) report a critical role for the transcriptional activator Eya4 in structuring the ET and in its positioning within the middle ear in mice. In Eya4 ${ }^{-/-}$mice, which are profoundly deaf, the ET epithelium was swollen and the medial osseous portion of the ET that opens into the middle-ear cavity was markedly narrowed and malpositioned at the anterior perimeter of the middle-ear cavity. The opening of the ET at the ostium was blocked by a polyp in $10 \%$ of Eya4 $4^{-/}$animals. These structural abnormalities appeared to predispose the mutant animals to the development of OME-like symptoms, including tympanic membrane retraction, inflammation of middle-ear cavity mucosa, and accumulation of middle-ear secretions (with or without bubbles). The results increase our knowledge of the genetic determinants of middle-ear development, and this animal model will be useful for the development of new therapies for genetic and possibly acquired forms of human OME.

\section{Table 1}

Comparison of OME signs in children and the Eya4-/ mouse

Structural variable
Eustachian tube
Middle-ear cavity
Craniofacial/palatal condition
TM condition
Hearing loss

\section{Structural variable}

Children

Short length, tube parallel to horizontal plane (increased likelihood of reflux) Ciliary dysfunction with inflammation; epithelial edema with occasional polyps; fluid-filled, with or without bubbles When defect is present, increased likelihood of OME Cloudy appearance with possible retraction 25 - to $35-\mathrm{dB}$ conductive loss

\section{Conclusions}

There are induced animal models of otitis media (see, for example, refs. 7-10). The introduction of human pathogenic organisms such as Steptococcus pneumoniae, Haemophilus influenzae, or Moraxella catharralis into the middle ear of an animal facilitates the investigation of clinically relevant infections. Experimental obstruction of the ET and introduction of inflammatory sub- 
stances into the middle ear have also been used to induce otitis media in mice (8). Genes that relate to mucin production and other functional aspects of the middle-ear cavity have also been identified $(11,12)$. In addition, the developmental anatomy of the middle ear and its conductive apparatus have been described for many common laboratory species (13-17).

This body of information, along with the identification of the current Eya4 ${ }^{-/-}$mutant mouse as a genetic model of OME (6), provides a rich database for future research on the treatment of chronic OME. This research might identify pharmacological approaches to reduce the long-term effects of effusions in the middle-ear space, or might seek to reduce or reverse cilia damage, polyp formation, and viscosity changes in the accumulated secretions. Treatments that maintain the patency of the ET may also be sought, and all such developments would represent important advances in the treatment of OME.

\section{Acknowledgments}

The National Institute on Deafness and Other Communication Disorders supports E. Lazaridis through training grant DC005363, while J.C. Saunders is supported by research award DC000710. The authors appreciate the comments of Mary Graham, Kirin Kennedy, Matthew Giampapola, and Michael Bilyk.

Address correspondence to: Evelyn Lazaridis, Department of Otorhinolaryngology: Head and Neck Surgery, University of Pennsylvania, 5 Ravdin-ORL, 3400 Spruce Street, Philadelphia, Pennsylvania 19104, USA. Phone: (215) 898-7504; Fax: (215) 898-7504; E-mail: elaz@mail.med.upenn.edu.

1. Rosenfeld, R.M., et al. 2004. Clinical practice guideline: otitis media with effusion. Otolaryngol. Head Neck Surg. 130:S95-S118.

2. Neto, W.C., et al. 2005. Otite média. FORL: Fundacao de Otorrinolaringologia website [In Portuguese]. http://www.forl.org.br/pdf/seminarios/ seminario_34.pdf.

3. Bluestone, C.R., and Klein, J.O. 2003. Otitis media and Eustachian tube dysfunction. In Pediatric otolaryngology. C. Bluestone, et al., editors. Saunders. Philadelphia, Pennsylvania, USA. 474-685.

4. Magnuson, B. 2001. Physiology of the Eustachian tube and middle ear pressure regulation. In Physiology of the ear. A.E. Jahn and J. Santos-Sacchi, editors. Singular. San Diego, California, USA. 75-99.

5. Wayne, S., et al. 2001. Mutations in the transcriptional activator EYA4 cause late-onset deafness at the DFNA10 locus. Hum. Mol. Genet. 195-200.

6. Depreux, F.F.S., et al. 2008. Eya4-deficient mice are a model for heritable otitis media. J. Clin. Invest.
118:651-658.

7. Zheng, Q.Y., Hardisty-Hughes, R., and Brown, S.D 2006. Mouse models as a tool to unravel the genetic basis for human otitis media. Brain Res. 1091:9-15.

8. Ryan, A.F., et al. 2006. Mouse models of induced otitis media [abstract]. Brain Res. 1091:3-8.

9. MacArthur, C.J., and Trune, D.R. 2006. Mouse models of otitis media. Curr. Opin. Otolaryngol. Head Neck Surg. 14:341-346.

10. Vicente, J., et al. 2007. Evolution of middle ear changes after permanent eustachian tube blockage. Arch. Otolaryngol. Head Neck Surg. 133:587-592.

11. Kerschner,J.E. 2007. Mucin gene expression in human middle ear epithelium. Laryngoscope. 117:1666-1676.

12. Elsheikh, M.N., and Malfouz, M.E. 2006. Up-regulation of MUC5AC and MUC5B mucin genes in nasopharygeal respiratory mucosa and selective upregulation of MUC5B in middle ear in pediatric otitis media [abstract]. Laryngoscope. 116:365-369.

13. Vrettakos, P.A., Dear, S.P., and Saunders, J.C. 1988. Middle-ear structure in the chinchilla: a quantitative study. Am. J. Otolaryngol. 9:58-67.

14. Cohen, Y.E., Bacon, C.K., and Saunders, J.C. 1992. Middle-ear development. III: Morphometric changes in the conducting apparatus of the Mongolian gerbil. Hear. Res. 62:187-193.

15. Zimmer, W.M., Rosin, D.F., and Saunders, J.C. 1994 Middle-ear development VI: Maturation of the rat conducting apparatus. Anat. Rec. 239:475-484.

16. Saunders, J.C., and Crumling, M.A. 2001. The structure and function of the outer and middle ear of the mouse. In Handbook of mouse anditory research: from behavior to molecular biology. J. Willott, editor. CRC Press. New York, New York, USA. 99-115.

17. Thomas, J.P., and Walsh, E.J. 1990. Postnatal development of the middle ear: Areal ratios in kittens. Otolaryngol. Head Neck Surg. 103:427-435.

\title{
Revisiting oxidative damage in ALS: microglia, Nox, and mutant SOD1
}

\author{
Séverine Boillée and Don W. Cleveland
}

Ludwig Institute for Cancer Research, Department of Medicine, and Department of Neuroscience, UCSD, La Jolla, California, USA.

\begin{abstract}
Mutation in superoxide dismutase-1 (SOD1) causes the inherited degenerative neurological disease familial amyotrophic lateral sclerosis (ALS), a non-cell-autonomous disease: mutant SOD1 synthesis in motor neurons and microglia drives disease onset and progression, respectively. In this issue of the JCI, Harraz and colleagues demonstrate that SOD1 mutants expressed in human cell lines directly stimulate NADPH oxidase (Nox) by binding to Rac1, resulting in overproduction of damaging ROS (see the related article beginning on page 659). Diminishing ROS by treatment with the microglial Nox inhibitor apocynin or by elimination of Nox extends survival in ALS mice, reviving the proposal that ROS mediate ALS pathogenesis, but with a new twist: it's ROS produced by microglia.
\end{abstract}

Amyotrophic lateral sclerosis (ALS) is the most common adult-onset motor neuron

Nonstandard abbreviations used: ALS, amyotrophic lateral sclerosis; Nox, $\mathrm{NADPH}$ oxidase; $\mathrm{O}_{2}{ }^{-}$, , superoxide; SOD1, superoxide dismutase-1.

Conflict of interest: The authors have declared that no conflict of interest exists.

Citation for this article: J. Clin. Invest. 118:474-478 (2008). doi:10.1172/JCI34613. disease. It is characterized by progressive paralysis and death, usually within 5 years, and no treatment substantially slows disease progression. The majority of ALS cases are sporadic, but among the familial cases of disease, mutations in the superoxide dismutase-1 (SOD1) gene are the most frequently identified. SOD1 catalyzes the conversion of superoxide $\left(\mathrm{O}_{2}{ }^{-}\right)$to $\mathrm{H}_{2} \mathrm{O}_{2}$, and elevation in redox stress has been proposed as a potential cause for motor neuron degeneration. Indeed, SOD1 mimetics have been reported to slow disease progression when administered at disease onset in ALS mice (1). Several hypotheses have been proposed in motor neuron degeneration, including damage within motor neurons and a non-cell autonomous aspect reflecting mutant SOD1-mediated damage within non-neuronal cells (2). Most of the studies supporting these hypotheses have been conducted in transgenic mice and rats in which expression of a mutant SOD1-encoding transgene recapitulates ALS-like disease, including muscle denervation and atrophy, glial activation, and motor neuron death. The importance of glial cell participation in disease was initially shown in chimeric mice in which mutant SOD1-expressing motor neurons 